\title{
On the Spectrum Occupancy Perception of Cognitive Radio Terminals in Realistic Scenarios
}

\author{
Miguel López-Benítez and Fernando Casadevall \\ Department of Signal Theory and Communications \\ Universitat Politècnica de Catalunya (UPC) \\ Barcelona, Spain \\ Email: \{miguel.lopez, ferranc\}@ tsc.upc.edu
}

\begin{abstract}
Cognitive radio terminals sense the spectrum to detect temporarily unoccupied spectrum gaps and transmit. The behavior of a network of cognitive radio terminals therefore depends on the spectrum occupancy perceived by each terminal at its local environment. In this context, this work explores (via empirical measurements) the spectrum occupancy that would be perceived by a cognitive radio terminal over a rich diversity of practical scenarios, including indoor environments as well as outdoor locations in high points and at the ground level (in open areas and between buildings). The impact of considering various locations on the spectral activity perceived by a cognitive radio terminal is determined, analyzed and quantified. The variety of considered scenarios provides a broader view and understanding of dynamic spectrum occupancy under different realistic scenarios of practical interest.
\end{abstract}

\section{INTRODUCTION}

The increasing spectrum demand growth experienced during the last years and the currently inefficient spectrum access policies have lead to a virtual spectrum scarcity problem, thus motivating the emergence of more dynamic spectrum access policies based on the Cognitive Radio (CR) technology. The basic underlying principle of $\mathrm{CR}$ is to allow unlicensed users to access in an opportunistic and non-interfering manner some licensed bands temporarily unoccupied by the licensed users. CR (secondary) terminals sense the spectrum in order to detect spectrum gaps not occupied by licensed (primary) users, usually referred to as spectrum holes or white spaces, and transmit. Secondary unlicensed transmissions are allowed following this operating principle as long as they do not cause excessive, harmful interference levels to the primary network.

It is clear that the behavior of a network of CR terminals, and consequently its impact on the primary network performance, depends on the spectrum occupancy perceived by each $\mathrm{CR}$ terminal at its local environment since it determines the local decisions on transmitting or refraining from transmitting. In the real world, the spectrum occupancy level that would be perceived by a CR terminal in any spectrum band can be quantitatively determined by means of field measurements. Measurements of the radio environment can provide valuable insights into actual and perceived spectrum usage. To the date, several spectrum measurement campaigns have already been performed in diverse locations and scenarios in order to determine the spectrum occupancy degree of allocated spectrum bands in real wireless communication systems [1-6]. However, most of previous spectrum occupancy studies are based on measurements performed in outdoor environments and more particularly in outdoor high points such as building roofs, balconies and towers. The main advantage of high points is that they provide direct line-of-sight to many kinds of primary transmitters and therefore enable a more accurate measurement of their actual spectral activity. Nevertheless, this scenario may not be representative of the spectrum occupancy that would be perceived by a CR terminal in many other interesting practical situations where the secondary user is not placed in a static high point (e.g., a mobile CR user communicating inside a building or while walking in the street between buildings). The measurement of real network activities in additional scenarios of practical significance is therefore required for an adequate and full understanding of the dynamic use of spectrum.

In this context, this work presents the results obtained in a spectrum measurement campaign performed over a rich diversity of practical scenarios in a densely populated urban environment in the city of Barcelona, Spain. The considered scenarios include not only high points but also indoor environments as well as outdoor locations at the ground level (in open areas and between buildings). The variety of considered measurement scenarios provides a broader view and understanding of dynamic spectrum use under different practical scenarios of interest. The aim of this work is to analyze, determine and quantify the impact of various locations on the spectral activity that would be perceived by a CR terminal with respect to that observed in an outdoor high point. This information will be very useful in the analysis of realistic scenarios as well as the development of realistic simulation tools.

\section{Measurement Setup and Methodology}

The measurement configuration employed in this work (see Figure 1) relies on a spectrum analyzer setup where different external devices have been added in order to improve the detection capabilities and hence obtain more accurate and reliable results. The design is composed of two broadband discone-type antennas covering the frequency range from 75 to $7075 \mathrm{MHz}$, a Single-Pole Double-Throw (SPDT) switch to select the desired antenna, several filters to remove undesired overloading (FM) and out-of-band signals, a low-noise preamplifier to enhance the overall sensitivity and thus the ability to detect weak signals, and a high performance spectrum 


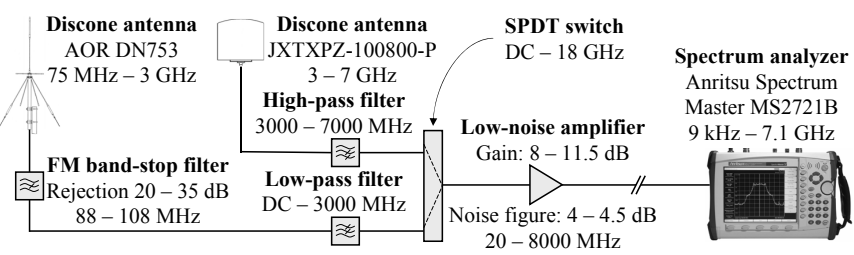

Fig. 1. Measurement setup employed in this study.

analyzer to record the spectral activity. The measurement setup and methodology employed in this work have been carefully designed based on the findings of the study presented in [7], where some important methodological aspects to be accounted for when evaluating spectrum occupancy in the context of $\mathrm{CR}$ are analyzed and discussed. A detailed description of the measurement setup design principles as well as the methodological procedures considered in this study can be found in [7].

\section{Measurement Scenarios}

The scenarios defined for this measurement campaign include not only outdoor but also indoor locations. Measurements in indoor locations provide information about the spectral activity that would be perceived by secondary users operating in indoor environments. Similarly, outdoor measurements give us some insights into the spectral activity that would be perceived by secondary users operating in outdoor environments, at various physical locations of practical interest. For the measurements in outdoor locations, three different kinds of scenarios have been considered, namely high points, narrow streets and open areas. Measurements in high points provide reliable information about the actual spectral occupancy patterns of several primary transmitters, while narrow streets and open areas give us an idea of the perception of secondary users moving within a urban environment with different levels of radio propagation blocking.

For indoor experiments, the measurement equipment was placed inside an urban building, in the middle floor of a three-floor building belonging to the Department of Signal Theory and Communications of the Universitat Politècnica de Catalunya (UPC), Barcelona, Spain. For outdoor high point measurements, the equipment was placed in the roof of the same building (latitude: $41^{\circ} 23^{\prime} 20^{\prime \prime}$ north; longitude: $2^{\circ}$ 6' 43" east; altitude: 175 meters). The selected place is a strategic location with direct line-of-sight to several transmitting stations located a few tens or hundreds of meters away from the antenna and without buildings blocking the radio propagation. This strategic location enabled us to accurately measure the spectral activity of, among others, TV and FM broadcast stations, several nearby base stations for cellular mobile communications and a military head quarter as well as some maritime and aeronautical transmitters due to the relative proximity to the harbor and the airport. For measurements in narrow streets and open areas, the measurement equipment was moved within the UPC's Campus Nord. The different geographical locations considered in this measurement campaign are illustrated in Figure 2 and described in Table I.

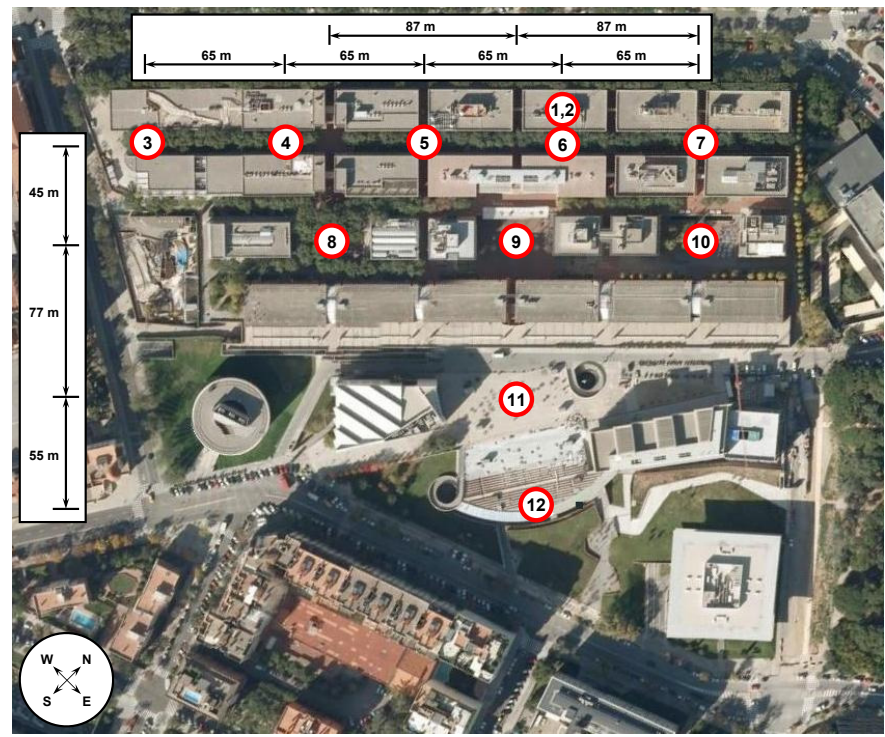

Fig. 2. Measurement locations in urban environment.

TABLE I

DESCRIPTION OF THE MEASUREMENT LOCATIONS.

\begin{tabular}{|c|c|}
\hline Measurement point & Environment \\
\hline 1 & Outdoor high point (building roof) \\
\hline 2 & Indoor (building room) \\
\hline $3-7$ & Outdoor at ground level in narrow streets \\
\hline $8-10$ & Outdoor at ground level between buildings \\
\hline $11-12$ & Outdoor at ground level in open areas \\
\hline
\end{tabular}

\section{SPeCtRum OCCUPANCY Results}

\section{A. Occupancy Metrics}

The occupancy level of various spectrum bands is quantified in this work in terms of the duty cycle. This metric is defined as the fraction of time that a band is measured as occupied and represents the average degree of spectrum utilization within certain time and frequency spans. To compute the duty cycle, the presence or absence of a licensed signal needs to be determined for the captured power samples. In other words, for each captured sample it is necessary to determine whether the sample corresponds to a licensed signal sample or a noise sample. To this end, an energy detection-based scheme is employed. Energy detection compares the received signal energy in a certain frequency band to a predefined decision threshold. If the signal energy lies above the threshold, a licensed signal is declared to be present. Otherwise, the measured channel is supposed to be idle. Based on the spectrum analyzer's power measurements, the duty cycle is computed as the fraction of recorded samples lying above a predefined decision threshold. The decision threshold is set according to the Probability of False Alarm 1\% (PFA 1\%) criterion as explained in [7].

\section{B. Comparison of Location 1 and Location 2}

This section presents and analyzes the results obtained in a urban indoor environment (location 2) taking as a reference the results obtained in an outdoor high point (location 1). The 
aim of this section is to determine the impact of considering an indoor environment on the spectral activity perceived by a secondary user with respect to that observed in an outdoor high point. Although the measurement conditions in both cases were identical, the time instants were different (i.e., both locations were not measured simultaneously). This circumstance introduces some random component in the obtained results since different transmissions were present in each case. However, it is worth noting that the aim of this section is not to characterize the instantaneous spectrum occupancy in the time domain but the average occupancy from a statistical point of view. For sufficiently long measurement periods as those considered in this study ( 24 hours), the impact of different instantaneous transmissions is averaged and the obtained average duty cycles can be considered as a representative indication of the spectral activity in the bands under study.

From a qualitative point of view, the results obtained in location 2 follow the same trend as in location 1, with higher occupancy rates at lower frequencies (a detailed analysis of the occupancy results obtained for location 1 can be found in $[8,9])$. As it can be appreciated in Table II, the average spectrum occupancy is moderate below $1 \mathrm{GHz}$ and very low above $1 \mathrm{GHz}$. The significantly lower average occupancy rates observed in Table II for the indoor location can be explained by the fact that most of wireless transmitters are located outdoor and the propagation loss due to outdoor-indoor signal penetration leads to lower signal strengths in the indoor scenario, which in turn results in lower occupancy rates. In principle, the lower average duty cycles obtained for the indoor case suggest the existence of a higher amount of free spectrum. However, this result should be interpreted carefully, taking into account the specific circumstances of particular bands.

To analyze the impact of the indoor location on the observed occupancy rate for various specific bands, it is convenient to distinguish four different possible cases according to the location of transmitters and receivers, as shown in Table III. Based on this classification, the results for various bands of interest are shown in Figure 3. Notice that for certain bands the classification is not straightforward. For example, in the downlink direction of cellular mobile communication systems the receivers are mobile users that may be located indoor and outdoor simultaneously. In practice, it is not possible to reliably determine the location of every transmitter and receiver operating in a certain band, which results in some uncertainty. In spite of that, some general trends can be inferred from the results shown in Figure 3.

For bands allocated to systems where the transmitters are always outdoor (cases I/II), the indoor duty cycles are in general notably lower, as expected, due to the outdoor-indoor signal penetration loss. In case I (systems with outdoor receivers) the lower indoor occupancy rates indicate the availability of more free spectrum, since an indoor secondary user transmitting in channels sensed as free would not cause harmful interference to primary outdoor receivers. However, in case II (systems with indoor receivers) the lower indoor duty cycles do not necessarily imply the existence of more white spaces, since in
TABLE II

AVERAGE DUTY CYCLE STATISTICS IN LOCATIONS 1 AND 2.

\begin{tabular}{|c|c|c|c|c|c|c|}
\hline \multirow{2}{*}{$\begin{array}{c}\text { Frequency } \\
\text { (MHz) }\end{array}$} & \multicolumn{7}{|c|}{ Average duty cycle (\%) } \\
\cline { 2 - 5 } & Loc.1 & Loc.2 & Loc.1 & Loc.2 & Loc.1 & Loc.2 \\
\hline $75-1000$ & 42.00 & 33.70 & \multirow{2}{*}{31.02} & 21.54 & & \\
\hline $1000-2000$ & 13.30 & 1.94 & & & \\
\hline $2000-3000$ & 3.73 & 1.63 & & & \multirow{2}{*}{17.78} & 12.10 \\
\hline $3000-4000$ & 4.01 & 1.44 & & & \\
\hline $4000-5000$ & 1.63 & 1.09 & \multirow{2}{*}{2.75} & 1.39 & & \\
\hline $5000-6000$ & 1.98 & 1.34 & & & & \\
\hline $6000-7075$ & 1.78 & 1.38 & & & & \\
\hline
\end{tabular}

TABLE III

CASES CONSIDERED IN Figure 3.

\begin{tabular}{|c|c|c|}
\hline Case & Transmitter location & Receiver location \\
\hline I & Outdoor & Outdoor \\
\hline II & Outdoor & Indoor \\
\hline III & Indoor & Outdoor \\
\hline IV & Indoor & Indoor \\
\hline
\end{tabular}

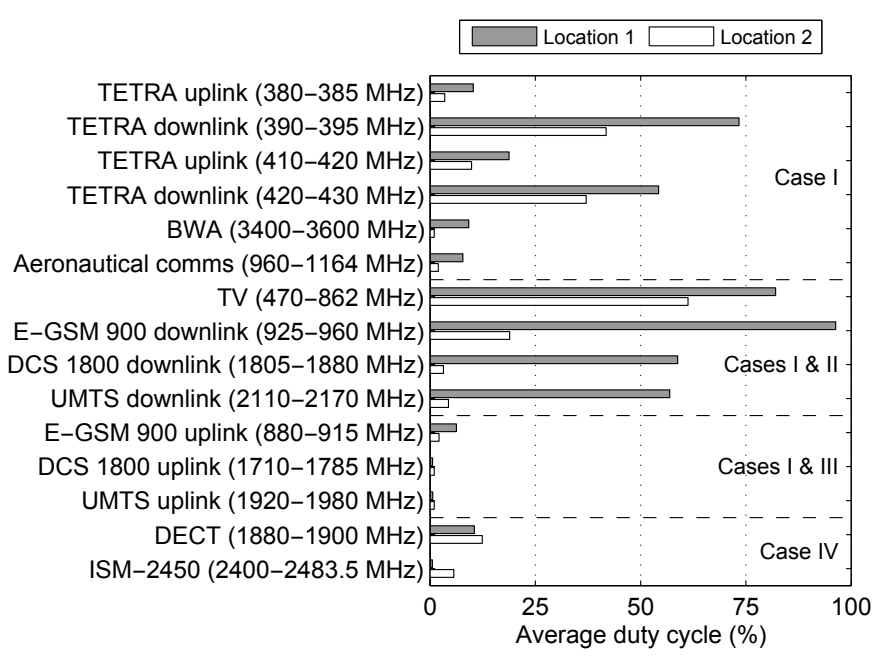

Fig. 3. Average duty cycle statistics in locations 1 and 2 for specific bands.

this case transmitting in a channel sensed as unoccupied could potentially result in interference to primary indoor receivers.

For bands allocated to systems with indoor transmitters (cases III/IV), in general the average duty cycles tend to be higher in the indoor location (with some exceptions as the EGSM 900 uplink band, which might be due to the presence of outdoor transmitters). Following a similar argument, in case IV (systems with indoor receivers) this indicates the availability of a lower amount of free spectrum, while it could not be necessarily the situation in case III (systems with outdoor receivers). In any case, the differences observed in this experiment between outdoor and indoor occupancy rates in cases III/IV are not as significant as in cases I/II.

In summary, although average duty cycles tend to be lower in indoor locations, this does not necessarily indicate the existence of more free spectrum. The particular circumstances of the sensed bands and the features of the systems operating over them need to be carefully considered before declaring a band as truly available for secondary usage; otherwise, harmful interference could be caused to the primary licensed system. 
Based on the previous results, it can be stated that the output of spectrum sensing procedures is not enough to declare a band as truly available for secondary access. Some additional techniques may be required such as, for example, sensing both the uplink and downlink directions of FDD-based systems in order to guarantee that the channel can be accessed opportunistically, or employing signal processing techniques as the one described in [9] in order to determine the signal standard present in a certain band before deciding whether a band can be accessed without inducing harmful interference.

Another aspect of practical interest is stressed by the results obtained in the $75-235 \mathrm{MHz}$ band, where the average duty cycle was significantly lower in the outdoor location $(48.43 \%)$ than in the indoor location $(66.56 \%)$, despite that most systems operating in these bands are located outdoor. As observed in Figure 4(a), this difference can be ascribed to a higher noise floor above the energy decision threshold in the indoor experiment (high noise levels may be interpreted as primary signals when energy detection is employed since it is not able to differentiate between intended primary transmissions and external noise sources). Notice that in the FM band (88$108 \mathrm{MHz}$ ), where a FM band stop filter is employed, the noise floor is lower than in the rest of bands, which indicates that the higher noise floor might be due to external noise sources (e.g., out-of-band transmissions, ambient noise or man-made noise). Man-made noise sources include emissions from electric motors (industrial equipments, trains, buses, etc.), Alternating Current (AC) power systems (consumer products, lighting systems, etc.), microwave ovens and spark plugs of automotive ignition systems, among many others [10, 11]. Several measurements of the environmental noise floor have shown that the most significant man-made noise component is observed at frequencies below $200 \mathrm{MHz}[12,13]$. At higher frequencies (above $1 \mathrm{GHz}$ ) studies of environmental noise have shown very low levels of noise except for emissions from microwave ovens centered at $2450 \mathrm{MHz}$ [14, 15]. Since manmade noise presents important variations with the environment [12], this could explain the differences observed between locations 1 and 2 in the 75-235 MHz band. To verify this point, the indoor experiment was repeated after deactivating most of the identified potential man-made noise sources in the vicinity of the measurement equipment (computers, air conditioning systems and two racks of computing servers). In this case a maximum measurement period of 4 hours was possible before noise sources became active again. The obtained results are shown in Figure 4(b). As it can be observed, the noise floor in this case is virtually flat, indicating that man-made noise sources have been removed, and the obtained average duty cycle is equal to $8.45 \%$, showing that the actual occupancy rate of this band is actually lower in indoor environments, as expected. From a practical point of view, these results show how external noise sources can alter the spectrum occupancy perception of CR terminals. In this case, a CR terminal would incorrectly infer a higher level of spectral activity and thus a lower amount of available spectrum opportunities. This indicates the need to employ more sophisticated spectrum sensing algorithms than the energy detection scheme considered in this study as well as some additional techniques as in [9] in order to minimize the waste of spectrum opportunities.

\section{Comparison of Location 1 and Locations 3-12}

This section presents and analyzes the results obtained in urban narrow streets and open areas (locations 3-12) taking as a reference the results obtained in an outdoor high point (location 1). The aim of this section is to determine the impact of considering different outdoor locations at the ground level on the spectral activity perceived by a secondary user with respect to that observed in an outdoor high point. The locations under study in this section can be considered as a representative scenario for secondary mobile users communicating while walking on the street in a urban environment.

As in section IV-B, each location has been measured at a different time instant. As mentioned above, the random component introduced by the presence of different transmissions at different times could be averaged by considering a sufficiently long measurement period. However, since the presence of an operative was required in the measurements, periods of 24 hours as in location 1 were infeasible and were therefore shortened to 1 hour in locations 3-12. To reduce the impact of random components and make the results of locations 1 and 3-12 comparable, the average duty cycle $\Psi_{k}$ obtained in locations $k=3,4, \ldots, 12$ (based on the samples captured during a 1-hour interval), have been normalized by the average duty cycle $\Psi_{1}$ obtained for location 1 when considering the samples corresponding to the same 1-hour time intervals. The normalized average duty cycle for location $k$ is thus obtained as $\bar{\Psi}_{k}=\Psi_{k} / \Psi_{1}$. This procedure reduces the randomness of the obtained results and enables a fairer comparison between the outdoor high point and the rest of outdoor locations.

For most of the bands and locations measured in this experiment the obtained normalized average duty cycle is lower than one, meaning that the average duty cycle measured in different locations at the ground level is in general lower than in high points. This is a consequence of the radio propagation blocking caused by buildings and other obstacles: under nonline-of-sight conditions, the direct ray (i.e., the strongest signal component) is lost; only multi path propagation components attenuated by reflection, refraction and diffraction are received, thus resulting in lower received signal levels and therefore in lower average duty cycles. From a practical point of view, this indicates that a secondary user at the ground level would perceive an amount of white space higher than that predicted by measurements performed in high points. Nevertheless, it is worth highlighting that this should be interpreted carefully, taking into account the specific circumstances of each band. In the following, some particular bands of interest are discussed. The obtained results are shown in Figure 5.

Figures 5(a), 5(b), 5(c) and 5(d) show the spatial distribution of the normalized average duty cycle for the TV, UMTS downlink, E-GSM 900 downlink and DCS 1800 downlink bands, respectively. The common feature of these bands is that the transmitters are located outside the region under 
study. In the TV band, it can be clearly appreciated that the normalized average duty cycle is lower in closed regions. Thus, in locations 4 and 6 , where radio propagation blocking caused by buildings is more intense, its value is lower than in other less closed regions such as locations 3,5 and 7. A similar trend is observed for UMTS, E-GSM 900 and DCS 1800 downlink bands. In the two last cases, however, location 5 constitutes an exception, which could be explained by the different relative position of transmitters and by the fact that the same physical scenario may result in very dissimilar propagation scenarios at different frequencies. Comparing locations 8, 9 and 10, the deepest region (location 9) exhibits the lowest normalized average duty cycle in the case of $\mathrm{TV}$, as expected, but the highest value in the case of the cellular mobile communication bands, which could be explained by the use of micro-cells and repeaters in shadowed regions as location 9. Regarding locations 11 and 12, it is interesting to note that a higher spectral activity level was recorded in location 11 despite the presence of some surrounding buildings with respect to the open region in location 12 . The detection by the measurement equipment of some additional signal components reflected in such buildings could explain the recording of higher activity levels in a less open region. Although lower duty cycles have been observed at the ground level in the TV, UMTS, E-GSM and DCS 1800 downlink bands, it is worth noting that this does not necessarily imply the existence of more opportunities for secondary access. As a matter of fact, some faded primary signals might be undetected at the ground level due to blocking buildings and other obstacles, in which case an exceptionally harmful interference would be caused to the intended primary receivers, who in these bands would be operating in the proximity of the secondary users. These experimental results highlight the importance of detection sensitivity in secondary networks and suggest the need of some additional techniques as mentioned in section IV-B.

In the previous bands, where the transmitters were outside the region under study, the results have shown some general occupancy trends. For other bands with transmitters operating inside the region under study, however, no particular occupancy trends have been observed. In this case, the obtained results might depend not on the actual spectral usage of such bands but rather on the random and fluctuating geographical distribution of transmitters inside the considered region.

\section{CONCLUSION}

The behavior of a network of cognitive radio terminals depends on the spectrum occupancy perceived by each terminal at its local environment. The spectrum measurement campaign carried out in this work over a rich diversity of practical scenarios indicates that the spectral activity perceived by a cognitive radio terminal in realistic urban scenarios strongly depends on the user location, with significant variations even in physical areas as reduced as the one considered in this study $(\approx 180 \mathrm{~m} \times 260 \mathrm{~m})$. This indicates that CR terminals operating within the same region may behave in a very dissimilar way. The dependence of the perceived spectral activity with the user location along with the presence of external noise sources (e.g., man-made noise sources) altering the observed spectrum occupancy suggest the need for sophisticated spectrum sensing methods as well as some additional techniques in order to guarantee an accurate spectrum occupancy detection and minimize the waste of spectrum opportunities while avoiding harmful interference to primary licensed systems.

\section{ACKNOWLEDGMENT}

The authors wish to acknowledge the activity of the Network of Excellence in Wireless COMmunications NEW$\mathrm{COM}++$ of the European Commission (contract n. 216715) that motivated this work. The support from the Spanish Ministry of Science and Innovation (MICINN) under FPU grant AP2006-848 is hereby acknowledged.

\section{REFERENCES}

[1] M. A. McHenry et al., "Spectrum occupancy measurements," Shared Spectrum Company, Tech. Rep., Jan 2004 - Aug 2005, available at: http://www.sharedspectrum.com.

[2] A. Petrin and P. G. Steffes, "Analysis and comparison of spectrum measurements performed in urban and rural areas to determine the total amount of spectrum usage," in Proc. Int'l Symp. on Advanced Radio Technologies (ISART 2005), Mar. 2005, pp. 9-12.

[3] O. Holland, P. Cordier, M. Muck, L. Mazet, C. Klock, and T. Renk, "Spectrum power measurements in $2 \mathrm{G}$ and $3 \mathrm{G}$ cellular phone bands during the 2006 Football World Cup in Germany," in Proc. 2nd IEEE Int'l Symp. on New Frontiers in Dynamic Spectrum Access Networks (DySPAN 2007), Apr. 2007, pp. 575-578.

[4] R. I. C. Chiang, G. B. Rowe, and K. W. Sowerby, "A quantitative analysis of spectral occupancy measurements for cognitive radio," in Proc. IEEE 65th Vehicular Technology Conf. (VTC 2007 Spring), Apr. 2007, pp. 3016-3020.

[5] M. Wellens, J. Wu, and P. Mähönen, "Evaluation of spectrum occupancy in indoor and outdoor scenario in the context of cognitive radio," in Proc. Second Int'l Conf. on Cognitive Radio Oriented Wireless Networks and Communications (CrowCom 2007), Aug. 2007, pp. 1-8.

[6] M. H. Islam, C. L. Koh, S. W. Oh, X. Qing, Y. Y. Lai, C. Wang, Y.-C. Liang, B. E. Toh, F. Chin, G. L. Tan, and W. Toh, "Spectrum survey in Singapore: Occupancy measurements and analyses," in Proc. 3rd Int'l Conf. on Cognitive Radio Oriented Wireless Networks and Communications (CrownCom 2008), May 2008, pp. 1-7.

[7] M. López-Benítez and F. Casadevall, "Methodological aspects of spectrum occupancy evaluation in the context of cognitive radio," in Proc. 15th European Wireless Conf. (EW 2009), May 2009, pp. 199-204.

[8] M. López-Benítez, A. Umbert, and F. Casadevall, "Evaluation of spectrum occupancy in Spain for cognitive radio applications," in Proc. IEEE 69th Vehicular Technology Conf. (VTC 2009 Spring), Apr. 2009, pp. 1-5.

[9] M. López-Benítez, F. Casadevall, A. Umbert, J. Pérez-Romero, J. Palicot, C. Moy, and R. Hachemani, "Spectral occupation measurements and blind standard recognition sensor for cognitive radio networks," in Proc. 4th Int'l Conf. on Cognitive Radio Oriented Wireless Networks and Communications (CrownCom 2009), Jun. 2009, pp. 1-9.

[10] E. N. Skomal, Man-made radio noise. Van Nostrand Reinhold, 1978.

[11] R. J. Matheson, "Measurements of electromagnetic noise radiated from automotive ignition systems," National Telecommunications and Information Administration (NTIA), Tech. Rep. 80-54, Nov. 1980.

[12] A. D. Spaulding and R. T. Disney, "Man-made radio noise, part 1 : Estimates for business, residential, and rural areas," Office of Telecommunications (OT), Tech. Rep. 74-38, Jun. 1974.

[13] R. J. Achatz and R. A. Dalke, "Man-made noise power measurements at VHF and UHF frequencies," National Telecommunications and Information Administration (NTIA), Tech. Rep. 02-390, Dec. 2001.

[14] R. Dalke, R. Achatz, Y. Lo, P. Papazian, and G. Hufford, "Measurement and analysis of man-made noise in VHF and UHF bands," in Proc. Wireless Communications Conf., Aug. 1997, pp. 229-233.

[15] P. Constantinou, D. Apostolakis, and M. Katsikis, "Man made noise measurements," in Proc. 41st IEEE Vehicular Technology Conf. (VTC 1991), May 1991, pp. 475-476. 

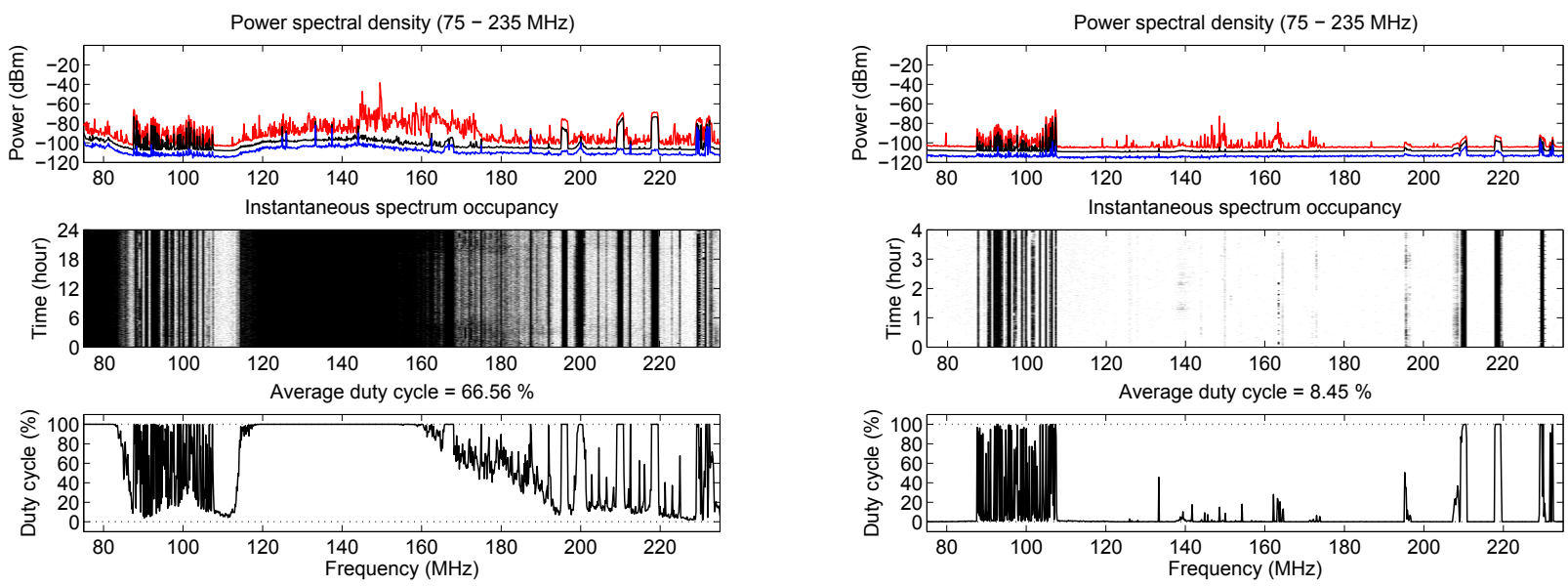

(a)

(b)

Fig. 4. Spectrum occupancy in location 2 (75-235 MHz): (a) In the presence of man-made noise, (b) Without man-made noise. Upper graphs show the power spectral density in maximum, average and minimum values. Middle graphs show the time evolution of the instantaneous spectrum occupancy (white and black colors indicate the absence and presence of primary signals, respectively). Lower graphs show the duty cycle as a function of the frequency.

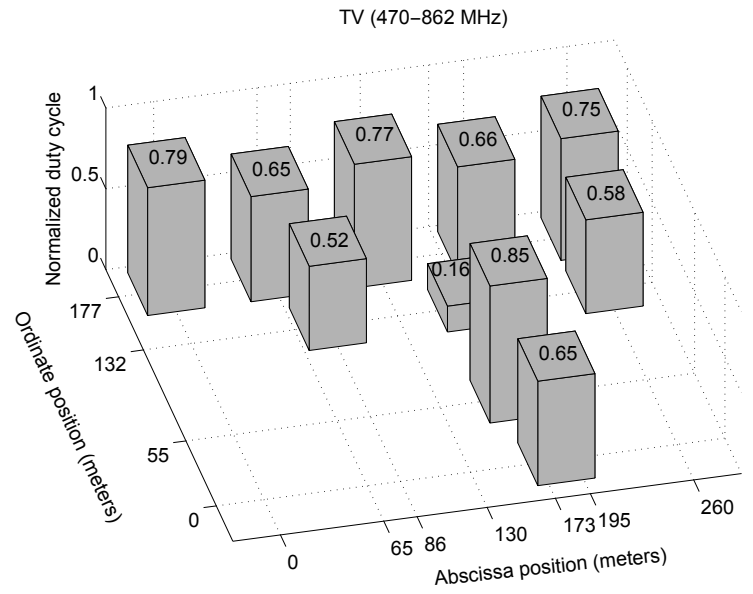

(a)

E-GSM 900 downlink $(925-960 \mathrm{MHz})$

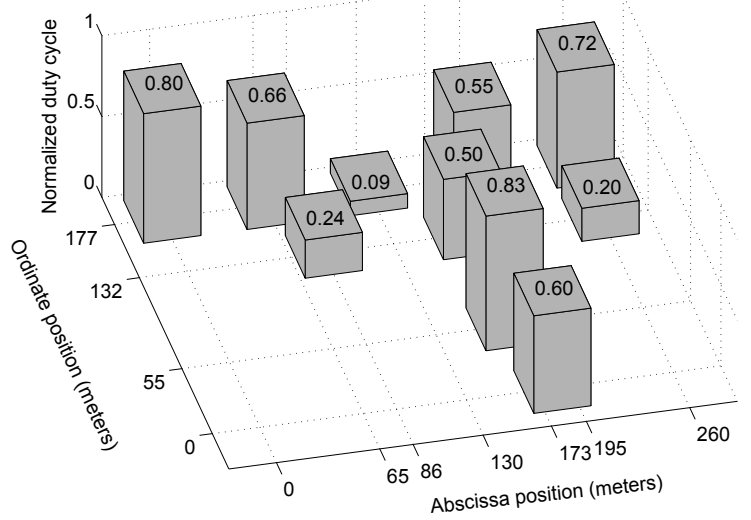

(c)
UMTS downlink (2110-2170 MHz)

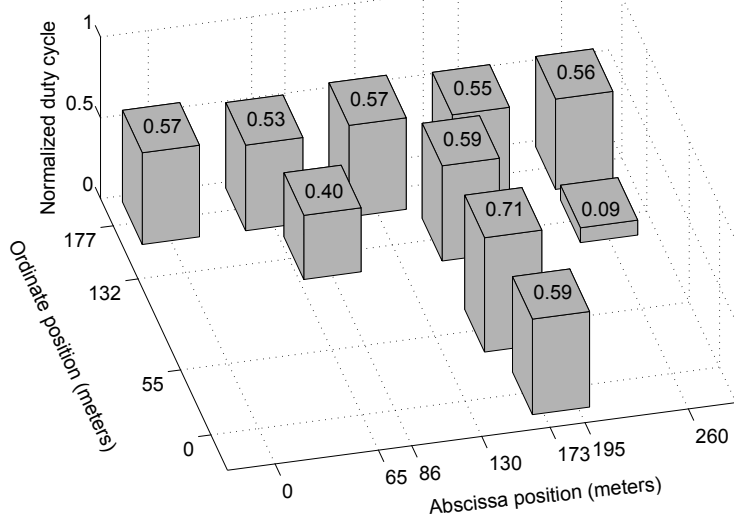

(b)

DCS 1800 downlink (1805-1880 MHz)

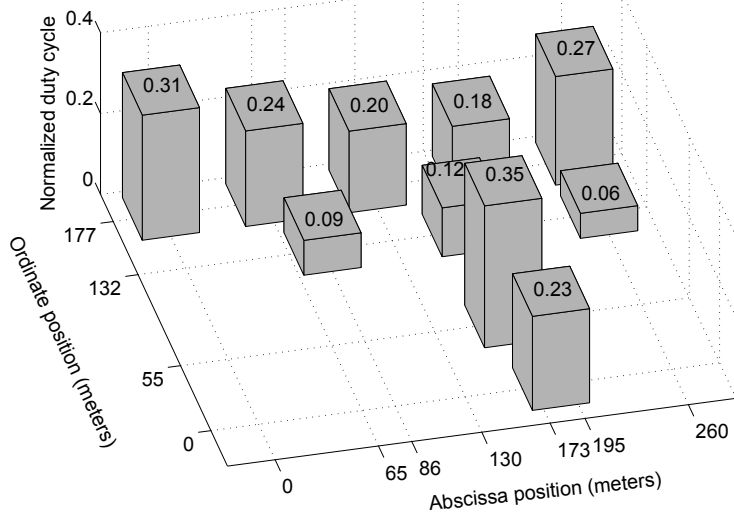

(d)

Fig. 5. Normalized average duty cycle statistics in locations 3 to 12 for specific bands: (a) TV (470-862 MHz), (b) UMTS downlink (2110-2170 MHz), (c) E-GSM 900 downlink (925-960 MHz), and (d) DCS 1800 downlink (1805-1880 MHz). From left-to-right and up-to-down, the positions of the bars in each graph correspond to the physical locations of points 3-12 (Figure 2). 